DFTT $54 / 94$

SNUTH-94-109

hep-ph/9411219

\title{
Atmospheric Neutrino Problem in Maximally-Mixed Three Generations of Neutrinos
}

\author{
C. Giunti ${ }^{\mathrm{a} *}$, C. W. Kim ${ }^{\mathrm{a}, \mathrm{b} \dagger}$ and J. D. Kim ${ }^{\mathrm{b} \ddagger}$ \\ ${ }^{a}$ INFN, Sezione di Torino and Dipartimento di Fisica Teorica, Università di Torino, \\ Via P. Giuria 1, 10125 Torino, Italy \\ ${ }^{\mathrm{b}}$ Department of Physics and Center for Theoretical Physics, \\ Seoul National University, Seoul, Korea
}

(March 1995)

\begin{abstract}
Motivated by the indication that both the atmospheric and the solar neutrino puzzles may simultaneously be solved by (vacuum as well as matter-induced resonant) oscillations of two generations of neutrinos with large mixing, we have analyzed the data on the atmospheric and solar neutrinos assuming that all three neutrinos are maximally mixed. It is shown that the values of $\Delta m^{2}$ obtained from the two-generation analyses are still valid even in the three-generation scheme, i.e. the two puzzles can be solved simultaneously if $\Delta m_{31}^{2} \simeq 10^{-2} \mathrm{eV}^{2}$ for the atmospheric neutrinos and $\Delta m_{21}^{2} \simeq 10^{-10} \mathrm{eV}^{2}$ for solar neutrinos in the maximally mixed three-generation scheme.
\end{abstract}

Typeset using REVTEX

*E-mail address: GIUNTI@TO.INFN.IT.

${ }^{\dagger}$ On leave of absence from The Johns Hopkins University, Baltimore, Maryland 21218, USA. E-mail address: CWKIM@JHUVMS.HCF.JHU.EDU.

${ }^{\ddagger}$ E-mail address: JDKIM@PHYB.SNU.AC.KR. 


\section{INTRODUCTION}

One of the several outstanding issues that we have faced so long in particle physics is that of neutrino masses and mixing (see, for example, Refs. [1,2]). Studies of solar neutrinos have been very promising for some time and already we have preliminary indications for positive answers [3-6]. In this case, however, one has to rely on the solar model [7], which is as good as it can be but it may not be, as yet, perfect. For this reason, many suggestions have been proposed on solar model-independent ways of analyzing solar neutrino data when more data will become available in the future $[8,9]$.

Another prominent source of neutrinos that has been investigated in the past is atmospheric neutrinos [10-16]. In this case, although the absolute fluxes of $\nu_{\mu}\left(\bar{\nu}_{\mu}\right)$ and $\nu_{e}\left(\bar{\nu}_{e}\right)$ are uncertain up to 30\% [17], if one takes the ratio of neutrino-induced muon and electron events both for data and MC simulations (in fact the ratio of the ratios), the results are almost model independent with errors of about $5 \%$ [17]. In this sense, the atmospheric neutrinos may be better suited for extracting information on the neutrino parameters such as mass and mixing angle.

Recently the Kamiokande collaboration [12] has analyzed more than 200 events with energies in the multi-GeV range and updated the previous sub-GeV data. Based on these data they have concluded that both data can be explained by either $\nu_{e}-\nu_{\mu}$ or $\nu_{\mu}-\nu_{\tau}$ oscillations. However, they have used explicitly the two generation formulations. The results of their analyses under the assumption that the anomalies are due to neutrino oscillations are summarized as being consistent with $\sin ^{2}(2 \vartheta) \simeq 1$ and $\Delta m^{2} \simeq 10^{-2}$, where $\vartheta$ and $\Delta m^{2}$ are, respectively, the $\nu_{e}-\nu_{\mu}$ mixing angle and $m_{2}^{2}-m_{1}^{2}$, and the $\nu_{\mu}-\nu_{\tau}$ mixing angle and $m_{3}^{2}-m_{2}^{2}$ for the $\nu_{e} \leftrightarrows \nu_{\mu}$ and $\nu_{\mu} \leftrightarrows \nu_{\tau}$ interpretations. (Whenever we mention $\nu_{e}, \nu_{\mu}$ and $\nu_{\tau}$ we also infer the cases of $\bar{\nu}_{e}, \bar{\nu}_{\mu}$ and $\bar{\nu}_{\tau}$.)

We also recall that in the case of solar neutrinos there are basically two popular ways to explain the so-called anomaly (under the assumption that the SSM is correct) with neutrino oscillations, one with vacuum oscillations and the other with the MSW effect [18]. Both cases contain solutions with almost maximal mixing of the neutrinos involved [19,20]. It is to be stressed here that for reaching the respective conclusions in all these analyses two generations of neutrinos have been used.

Now we may ask an obvious and logical question: Do these results remain valid even when the third generation neutrino is included? After all, we do know that there exist three neutrinos associated with $e, \mu$ and $\tau$. However, a three-generation analysis of any phenomenon is known to be very complicated and tedious, and often it is hard to see the physics involved [21].

In this work, we analyze the atmospheric and solar neutrinos using the three generations of neutrinos that are maximally mixed to begin with. Although this is, admittedly, an extreme case, it is a very good approximation in the event of large neutrino mixings, and, moreover, the algebra and analysis of the data become very simple and the physics becomes transparent. Furthermore, this does not imply a loss of generality if mixings turn out to be reasonably large because the qualitative results still remain valid.

The three-generation neutrino oscillation with maximal mixing has also been discussed in the past [22] without taking into account the energy and distance dependence of the oscillating terms. In this case the survival probability for the solar neutrinos, $P\left(\nu_{e} \rightarrow \nu_{e}\right)$, is 
$1 / 3$ at all energies. In Ref. [22] also the atmospheric neutrino anomaly has been addressed by assuming that all $\Delta m_{i j}^{2}$ are in the range $(0.5-1.2) \times 10^{-2} \mathrm{eV}^{2}$.

Our main purpose in this paper is to see if and how the two generation results are modified when the third neutrino generation is introduced by taking into account the energy and distance dependence of the oscillating terms.

\section{MAXIMAL MIXING AMONG THREE NEUTRINOS}

We consider three neutrinos to be maximally mixed if their survival probabilities are all equal and for distances much larger than the oscillation lengths all the three survival probabilities average to $1 / 3$. This can be accomplished with the following mixing matrix $[23,24]$ :

$$
U=\frac{1}{\sqrt{3}}\left(\begin{array}{ccc}
1 & 1 & 1 \\
1 & \mathrm{e}^{4 \pi i / 3} & \mathrm{e}^{2 \pi i / 3} \\
1 & \mathrm{e}^{2 \pi i / 3} & \mathrm{e}^{4 \pi i / 3}
\end{array}\right) .
$$

The presence in $U$ of the cube roots of unity $1, \mathrm{e}^{2 \pi i / 3}, \mathrm{e}^{4 \pi i / 3}$ is necessary for $U$ to be unitary. It follows that in the maximal mixing case $\mathrm{CP}$ must be violated. Using the mixing matrix (2.1) we obtain the survival probabilities

$$
\begin{aligned}
P\left(\nu_{e} \rightarrow \nu_{e}\right) & =P\left(\nu_{\mu} \rightarrow \nu_{\mu}\right)=P\left(\nu_{\tau} \rightarrow \nu_{\tau}\right) \\
& =1-\frac{4}{9}\left(\sin ^{2} k_{21}+\sin ^{2} k_{31}+\sin ^{2} k_{32}\right),
\end{aligned}
$$

with

$$
k_{i j} \equiv \frac{1.27 L \Delta m_{i j}^{2}}{E} .
$$

Here $L, E$ and $\Delta m_{i j}^{2} \equiv m_{i}^{2}-m_{j}^{2}$ are in units of meter, $\mathrm{MeV}$ and $\mathrm{eV}^{2}$, respectively. On the other hand, the probabilities of transition between different neutrino flavors are not identical. Instead, we have

$$
\begin{aligned}
P\left(\nu_{\mu} \rightarrow \nu_{e}\right) & =P\left(\nu_{e} \rightarrow \nu_{\tau}\right)=P\left(\nu_{\tau} \rightarrow \nu_{\mu}\right) \\
& =1-\frac{4}{9}\left[\sin ^{2}\left(k_{21}+\frac{\pi}{3}\right)+\sin ^{2}\left(k_{31}-\frac{\pi}{3}\right)+\sin ^{2}\left(k_{32}+\frac{\pi}{3}\right)\right]
\end{aligned}
$$

and

$$
\begin{aligned}
P\left(\nu_{e} \rightarrow \nu_{\mu}\right) & =P\left(\nu_{\mu} \rightarrow \nu_{\tau}\right)=P\left(\nu_{\tau} \rightarrow \nu_{e}\right) \\
& =1-\frac{4}{9}\left[\sin ^{2}\left(k_{21}-\frac{\pi}{3}\right)+\sin ^{2}\left(k_{31}+\frac{\pi}{3}\right)+\sin ^{2}\left(k_{32}-\frac{\pi}{3}\right)\right]
\end{aligned}
$$

It is interesting to notice that the mixing matrix for maximal mixing is not unique. In fact, all the matrices which are obtained from $U$ by multiplying any number of rows or columns by a phase give the same oscillation probabilities (2.2), (2.4) and (2.5). Furthermore, $U^{\dagger}$ gives the same survival probabilities (2.2), but all the phases $\pi / 3$ in the transition probabilities (2.4) and (2.5) change sign. Since the definition of maximal mixing requires the survival probabilities (2.2), it follows that both $U$ and $U^{\dagger}$ give maximal mixing, but they produce two different phenomenologies for the transition probabilities. 


\section{ATMOSPHERIC NEUTRINOS}

As in many previous works, our study of atmospheric neutrinos involves the ratio $\left(\nu_{\mu}+\bar{\nu}_{\mu}\right) /\left(\nu_{e}+\bar{\nu}_{e}\right)$. In actual experimental situations, this ratio manifests as an observed ratio of muon-like and electron-like events. Although the issue of whether or not the observed ratio is indeed small compared with the Monte Carlo (MC) simulated ratio is still not completely settled [10-16], the Kamiokande group recently presented two sets of impressive data, one involving the electron and muon events in the sub-GeV energy range and the other in the multi-GeV energy range. The Kamiokande collaboration found [12]

$$
\mathrm{R}^{\exp } \equiv \frac{\mathrm{R}(\mu / e)_{\exp }}{\mathrm{R}(\mu / e)_{\mathrm{MC}}}= \begin{cases}0.60_{-0.05}^{+0.06} \pm 0.05 & \text { for the sub-GeV energy range }, \\ 0.57_{-0.07}^{+0.08} \pm 0.07 & \text { for the multi-GeV energy range },\end{cases}
$$

where $\mathrm{R}(\mu / e)_{\exp }$ and $\mathrm{R}(\mu / e)_{\mathrm{MC}}$ are the ratios of $\mu$-like and $e$-like events observed and calculated, respectively. In addition, they studied the dependence of the above ratios on the zenith-angle $\theta$ in the range $-1 \leq \cos \theta \leq 1$, divided in 5 bins. The zenith-angle dependence of $\mathrm{R}^{\exp }$ for the sub-GeV data is rather flat (see Fig.6 of Ref. [12]), whereas that of the multi$\mathrm{GeV}$ data appears to deviate from being flat (see Fig.4 of Ref. [12]). Our analysis will be based only on the Kamiokande data.

Let us consider the zenith-angle dependence of the Kamiokande data. For each bin $i$ $\left(i=1, \ldots, 5\right.$ for $\langle\cos \theta\rangle_{i=1, \ldots, 5}=-0.8,-0.4,0.0,0.4,0.8$, which correspond to the averaged distances $\left.\langle L\rangle_{i=1, \ldots, 5}=10,230,5,157,852,54,26 \mathrm{Km}\right)$, the number $N_{e i}$ of $e$-like events, the number $N_{\mu i}$ of $\mu$-like events and the ratio of ratios $\mathrm{R}_{i}$ are given by

$$
\begin{aligned}
& N_{e i}=\frac{\rho_{\mathrm{T}}}{\sqrt{\rho_{\mathrm{R}}}} N_{e i}^{\mathrm{MC}} P_{i}\left(\nu_{e} \rightarrow \nu_{e}\right)+\rho_{\mathrm{T}} \sqrt{\rho_{\mathrm{R}}} N_{\mu i}^{\mathrm{MC}} P_{i}\left(\nu_{\mu} \rightarrow \nu_{e}\right), \\
& N_{\mu i}=\frac{\rho_{\mathrm{T}}}{\sqrt{\rho_{\mathrm{R}}}} N_{e i}^{\mathrm{MC}} P_{i}\left(\nu_{e} \rightarrow \nu_{\mu}\right)+\rho_{\mathrm{T}} \sqrt{\rho_{\mathrm{R}}} N_{\mu i}^{\mathrm{MC}} P_{i}\left(\nu_{\mu} \rightarrow \nu_{\mu}\right), \\
& \mathrm{R}_{i} \equiv \frac{N_{\mu i} / N_{\mu i}^{\mathrm{MC}}}{N_{e i} / N_{e i}^{\mathrm{MC}}}=\rho_{\mathrm{R}} \frac{P_{i}\left(\nu_{\mu} \rightarrow \nu_{\mu}\right)+\rho_{\mathrm{R}}^{-1}\left(N_{\mu i}^{\mathrm{MC}} / N_{e i}^{\mathrm{MC}}\right)^{-1} P_{i}\left(\nu_{e} \rightarrow \nu_{\mu}\right)}{P_{i}\left(\nu_{e} \rightarrow \nu_{e}\right)+\rho_{\mathrm{R}}\left(N_{\mu i}^{\mathrm{MC}} / N_{e i}^{\mathrm{MC}}\right) P_{i}\left(\nu_{\mu} \rightarrow \nu_{e}\right)} .
\end{aligned}
$$

Here $N_{e i}^{\mathrm{MC}}$ and $N_{\mu i}^{\mathrm{MC}}$ are, respectively, the number of $e$-like and $\mu$-like events in the bin $i$ predicted by MC simulations, without neutrino oscillations, after passing through the same analysis chain as the data. The factors $\rho_{\mathrm{T}}$ and $\rho_{\mathrm{R}}$ are the normalization coefficients that take into account the systematic error of the $\mathrm{MC}$ calculation of the total number of $e$-like and $\mu$-like events $(30 \%)$ and the systematic error of the $\mathrm{MC}$ calculation of the $\mu / e$ ratio ( $9 \%$ for the sub-GeV data and $12 \%$ for the multi-GeV data), respectively. We inserted these two factors in Eqs.(3.2) and (3.3) in such a way that the ratio of ratios $R_{i}$ does not depend on $\rho_{\mathrm{T}}$, i.e., only the coefficient $\rho_{\mathrm{R}}$ appears in the expression (3.4) for the ratio of ratios as an overall multiplicative factor and the ratio $\left(N_{\mu i}^{\mathrm{MC}} / N_{e i}^{\mathrm{MC}}\right)$ is always multiplied by $\rho_{\mathrm{R}}$. As a consequence, the error of $\mathrm{R}_{i}$ does not depend on the systematic error of the $\mathrm{MC}$ calculation of the total number of $e$-like and $\mu$-like events, but only on the systematic error of the $\mathrm{MC}$ calculation of the $\mu / e$ ratio, which is much smaller. In Eqs.(3.2)-(3.4) we neglected the fact that not all $e$-like and $\mu$-like events are produced by $\nu_{e}\left(\bar{\nu}_{e}\right)$ and $\nu_{\mu}\left(\bar{\nu}_{\mu}\right)$, 
respectively. However, the purity of the $\nu_{e}$ and $\nu_{\mu}$ contributions to the selected $e$-like and $\mu$-like events is estimated by the Kamiokande Collaboration to be higher than $90 \%$. We wish to emphasize here that our three generation formalism takes into account simultaneously the two neutrino oscillation channels $\nu_{\mu} \leftrightarrows \nu_{e}$ and $\nu_{\mu} \rightarrow \nu_{\tau}$. This is not the case for two generation analyses, where the two oscillation channels are considered separately, leading to two possible interpretations and results, as done in Ref. [12].

Since we have $L \lesssim 13 \times 10^{3} \mathrm{Km}, E \gtrsim 0.2 \mathrm{GeV}$ and $\Delta m_{21}^{2} \simeq 10^{-10} \mathrm{eV}^{2}$ (as will be seen later from the solution of the solar neutrino problem), $k_{21}$ is well approximated by zero and, because of the assumed hierarchy $m_{3}>m_{2}>m_{1}$, we expect $k_{32} \simeq k_{31}$, so that the relevant transitions probabilities are

$$
P\left(\nu_{e} \rightarrow \nu_{\mu}\right)=P\left(\nu_{\mu} \rightarrow \nu_{e}\right)=P\left(\nu_{\mu} \rightarrow \nu_{\tau}\right)=P\left(\nu_{e} \rightarrow \nu_{\tau}\right)=\frac{4}{9} \sin ^{2} k_{32} .
$$

We obtained the values of $N_{e i}^{\mathrm{MC}}$ and $N_{\mu i}^{\mathrm{MC}}$ from the $\mathrm{MC}$ data for the $e$-like and $\mu$-like events presented in Fig.2 of Ref. [10] for the sub-GeV data and the MC data presented in Fig.3 of Ref. [12] for the multi-GeV data. The quantities $P_{i}\left(\nu_{e} \rightarrow \nu_{\mu}\right)$ and $P_{i}\left(\nu_{\mu} \rightarrow \nu_{e}\right)$ are the $\nu_{e} \rightarrow \nu_{\mu}$ and $\nu_{\mu} \rightarrow \nu_{e}$ transition probabilities averaged over the neutrino energy spectrum and the zenith-angle width of bin $i$. These probabilities depend on the value of $\Delta m_{32}^{2}$ and they are different because initial $\nu_{e}$ and $\nu_{\mu}$ have different energy spectra. These energy spectra are given in Fig.1 of Ref. [11] for the sub-GeV data and the MC data presented in Fig.2 of Ref. [12] for the multi-GeV data.

Before we present the results of our full analysis, we discuss the following simple but interesting possibility. As suggested by the zenith-angle distribution of the sub-GeV data and to some extent (within error bars) by the large-angle data points of the multi-GeV data with $i=1, \ldots, 4$, corresponding to $\langle\cos \theta\rangle$ from -0.8 to $0.4, \mathrm{R}^{\exp }$ is roughly consistent with being flat. This may be interpreted as due to washing out of the distance dependence in the argument of $\sin ^{2}\left(1.27 L \Delta m_{32}^{2} / E\right)$ (the averaged distance $\langle L\rangle$ ranges from $10,230 \mathrm{Km}$ to $26 \mathrm{Km}$ for the sub-GeV data and from 10,230 Km to $54 \mathrm{Km}$ for the multi-GeV data). That is, the oscillations are in the rapid oscillation region with $\sin ^{2}\left(1.27 L \Delta m_{32}^{2} / E\right) \simeq 1 / 2$ and $P\left(\nu_{\alpha} \rightarrow \nu_{\beta}\right) \simeq 2 / 9$ for $\alpha \neq \beta$. Under this assumption, Eq.(3.4) reduces to

$$
\mathrm{R}_{i}=\rho_{\mathrm{R}} \frac{1-\frac{1}{9}+\frac{2}{9} \rho_{\mathrm{R}}^{-1}\left(N_{\mu i}^{\mathrm{MC}} / N_{e i}^{\mathrm{MC}}\right)^{-1}}{1-\frac{1}{9}+\frac{2}{9} \rho_{\mathrm{R}}\left(N_{\mu i}^{\mathrm{MC}} / N_{e i}^{\mathrm{MC}}\right)} .
$$

Since we have $N_{\mu i}^{\mathrm{MC}} /\left.N_{e i}^{\mathrm{MC}}\right|_{i=1, \ldots, 5} \simeq 1.57$ for the sub-GeV data and $N_{\mu i}^{\mathrm{MC}} /\left.N_{e i}^{\mathrm{MC}}\right|_{i=1, \ldots, 4} \simeq$ $2.98,2.29,2.08,2.39$ for the multi-GeV data, and taking into account the systematic error of $\rho_{\mathrm{R}}$, we find $\mathrm{R}_{i=1, \ldots, 5}=0.83 \pm 0.04$ for the sub-GeV data and $\mathrm{R}_{i=1, \ldots, 4}=$ $0.62 \pm 0.04,0.70 \pm 0.05,0.74 \pm 0.05,0.69 \pm 0.04$ for the multi-GeV data. These values are to be compared with $\left\langle\mathrm{R}_{i}^{\text {exp }}\right\rangle=0.60_{-0.07}^{+0.08}$ for the average of the sub-GeV data and $\mathrm{R}_{i=1, \ldots, 4}^{\exp }=0.29_{-0.07}^{+0.12}, 0.46_{-0.13}^{+0.17}, 0.51_{-0.11}^{+0.15}, 0.63_{-0.16}^{+0.22}$ for the multi-GeV data. It appears that the idea of rapid oscillations is not too inconsistent with the observations.

Let us now discuss the results of a full analysis of the Kamiokande data taking into account the oscillation effect. For different values of $\Delta m_{32}^{2}$ we have fitted separately the 
sub-GeV data of the ratio of ratios presented in Fig.6 of Ref. [12] and the multi-GeV data of the number of $e$-like and $\mu$-like events presented in Fig.3 of Ref. [12]. For the fit of the sub$\mathrm{GeV}$ data we used the theoretical formula for the ratio of ratios given in Eq.(3.4) and for the fit of the multi-GeV data we used the theoretical formulas for the number of $e$-like and $\mu$-like events given in Eqs.(3.2) and (3.3). For each value of $\Delta m_{32}^{2}$ we have calculated the minimum value of the $\chi^{2}$ with respect to the normalization coefficients $\rho_{\mathrm{T}}$ and $\rho_{\mathrm{R}}$, whose difference from unity contributes to the $\chi^{2}$ with errors $\sigma\left(\rho_{\mathrm{R}}\right)=9 \%$ for the sub-GeV data and $\sigma\left(\rho_{\mathrm{T}}\right)=30 \%$, $\sigma\left(\rho_{\mathrm{R}}\right)=12 \%$ for the multi-GeV data. Some results are depicted in Fig.1 together with the experimental data. As can be seen in Fig.1, the sub-GeV data and multi-GeV data can be best fitted (solid lines) with $\Delta m_{32}^{2}=1.3 \times 10^{-2} \mathrm{eV}^{2}$ and $\Delta m_{32}^{2}=3.0 \times 10^{-2} \mathrm{eV}^{2}$, respectively. In Fig. 2 we have plotted the $\chi^{2}$ of the fits as functions of $\Delta m_{32}^{2}$. As mentioned already, the best fits of the sub-GeV (Fig.2a) and multi-GeV (Fig.2b) data are obtained for $\Delta m_{32}^{2}=1.3 \times 10^{-2} \mathrm{eV}^{2}\left(\chi^{2}=4.9\right.$ with 3 degrees of freedom $)$ and $\Delta m_{32}^{2}=3.0 \times 10^{-2} \mathrm{eV}^{2}$ $\left(\chi^{2}=8.1\right.$ with 7 degrees of freedom), respectively. The separate fits of the the sub-GeV and multi-GeV data are acceptable at 18\% CL and 32\% CL, respectively. The $90 \% \mathrm{CL}$ allowed range of $\Delta m_{32}^{2}$ is determined by $\chi^{2}<\chi_{\min }^{2}+2.7$, that gives $\Delta m_{32}^{2}>8.1 \times 10^{-3} \mathrm{eV}^{2}$ from the sub-GeV data and $2.6 \times 10^{-3} \mathrm{eV}^{2}<\Delta m_{32}^{2}<6.3 \times 10^{-2} \mathrm{eV}^{2}$ from multi-GeV data. We see that these allowed ranges are perfectly compatible. Fig.2c gives the $\chi^{2}$ of the combined fit of the sub-GeV and multi-GeV data. Here there is a minimum for $\Delta m_{32}^{2}=2.0 \times 10^{-2} \mathrm{eV}^{2}$, very close to the two independent best fits of the sub-GeV and multi-GeV data. Since $\chi_{\min }^{2}=13.8$ and we have 11 degrees of freedom, the fit is acceptable at $24 \%$ CL. As can be seen from Fig.2c, the simultaneous fit of both the sub-GeV and multi-GeV data allows to constrain the value of $\Delta m_{32}^{2}$ to lie in the interval $8.9 \times 10^{-3} \mathrm{eV}^{2}<\Delta m_{32}^{2}<5.6 \times 10^{-2} \mathrm{eV}^{2}$ at $90 \%$ CL. This result agrees well with those given in Ref. [12], obtained from two generation analyses. We wish to add here that the values of $\Delta m_{32}^{2}$ larger than $9.0 \times 10^{-3} \mathrm{eV}^{2}$ and larger than $2.0 \times 10^{-2} \mathrm{eV}^{2}$ are excluded at $90 \% \mathrm{CL}$ by the Kurchatov [25] and Gosgen [26] reactor neutrino oscillation experiments, respectively. If combined, we are left with a very restricted allowed range for the value of $\Delta m_{32}^{2}$, which is about $10^{-2} \mathrm{eV}^{2}$.

\section{SOLAR NEUTRINOS}

We now proceed to check if the three-generation maximally mixed scheme discussed so far is also capable of solving the solar neutrino problem. Since we assume $\Delta m_{31}^{2} \simeq \Delta m_{32}^{2} \gg$ $10^{-10} \mathrm{eV}^{2}$ as obtained in the atmospheric neutrino problem, we have, from Eqs.(2.4) and $(2.5)$,

$$
\frac{P\left(\nu_{e} \rightarrow \nu_{\mu}\right)+P\left(\nu_{e} \rightarrow \nu_{\tau}\right)}{2}=\frac{2}{9}+\frac{2}{9} \sin ^{2} k_{21}
$$

and, from Eq.(2.2),

$$
P\left(\nu_{e} \rightarrow \nu_{e}\right)=\frac{5}{9}-\frac{4}{9} \sin ^{2} k_{21}
$$

Note that the maximal value of the survival probability of $\nu_{e}$ is $(5 / 9)=0.56$. This implies that, since the Standard Solar Model predicts the event rate for the Gallium detectors to be $132_{-6}^{+9}$ SNU, the observed event rate must be 


$$
\Sigma_{\exp } \lesssim 80
$$

If the $\Sigma_{\text {exp }}$ turns out to be greater than the above, this maximal mixing model (as an explanation with vacuum oscillation) is ruled out (or $\Delta m_{32}^{2}$ and $\Delta m_{31}^{2}$ must take values such that $\sin ^{2} k_{32}$ and $\sin ^{2} k_{31}$ cannot be approximated by $1 / 2$ ). The latest average of the GALLEX [5] and SAGE [6] event rates is $\Sigma_{\exp }=74 \pm 9$ which is still consistent with Eq. (4.3).

The results of the calculations for the Kamiokande, Homestake and Gallium experiments are shown in Fig.3. These calculations are based on the standard procedure, with the neutrino fluxes predicted by the Bahcall and Pinsonneault SSM [27], the detector cross sections given in Refs. [7,28], and the probabilities given in Eqs.(4.1) and (4.2).

In the figures, the horizontal solid lines are the central values of the experiments with the dashed lines representing one standard deviation. The oscillating solid lines represent the theoretical values, with the dotted lines indicating one standard deviation resulting from the errors in the SSM. We can see that in the neighborhood of $\Delta m_{21}^{2} \simeq 10^{-10} \mathrm{eV}^{2}$ theory and experiments agree reasonably well, with the exception of the Gallium experiments, in which case an agreement is obtained roughly within two standard deviations. The allowed values close to $\Delta m_{21}^{2} \simeq 10^{-10} \mathrm{eV}^{2}$ are in agreement with those obtained by two-generation analyses $[22,19]$.

\section{CONCLUSIONS}

Assuming maximally-mixed three-generations of neutrinos, we have analyzed the recent data on atmospheric neutrinos obtained by the Kamiokande Collaboration, which presented a corresponding analysis in the two-neutrino scheme.

The study is motivated by the indications of possible large mixing angle solutions for both the atmospheric and solar neutrino problems. In particular, in the recent Kamiokande analysis of the atmospheric neutrino data, the best fit values of $\sin ^{2}(2 \vartheta)$ are shown to be almost unity. Both the sub-GeV and multi-GeV data can be explained with $\Delta m_{32}^{2} \simeq$ $2 \times 10^{-2} \mathrm{eV}^{2}$. In our three-generation case the range of values of $\Delta m_{32}^{2}$ agrees with that of the two-generation analyses, but the values of $\sin ^{2}\left(2 \vartheta_{\alpha \beta}\right)$ are $0.89(\alpha, \beta=e, \mu, \tau)$ because the maximal mixing angles are $35^{\circ}$ instead of $45^{\circ}$, which is the case in the two-generation schemes. We conclude therefore that, besides this difference, the addition of the third generation of neutrinos does not change the range of $\Delta m_{32}^{2}$ obtained in the two-generation analysis.

Our solutions are based on vacuum oscillations. For the range of $\Delta m_{32}^{2}$ that we obtained, the medium effects of the Earth are negligible. Downward-going neutrinos do not travel enough in matter and for upward-going neutrinos the large neutrino mixing and the value of $\Delta m_{32}^{2}$ make the MSW effect inoperative.

We have also checked the solar neutrino problem in the same scheme. One definite prediction of the maximally mixed three-generation model is that the observed event rate for the Gallium experiments cannot be larger than 80 SNU. With exception of the Gallium case, the scheme provides a good fit of the data, assuming vacuum oscillations with values of $\Delta m_{21}^{2} \simeq 10^{-10} \mathrm{eV}^{2}$, similar in magnitude to those obtained in the two-generation analyses. 
Therefore, we conclude that the maximally-mixed three generations of neutrinos are capable of explaining both the current atmospheric and solar neutrino problems (though marginally in the case of the Gallium solar neutrino experiments). Improved data in the future will decide the fate of this scheme.

Finally, the obvious question is: Is it possible for completely non-degenerate neutrinos (with the standard mass hierarchy $m_{1} \ll m_{2} \ll m_{3}$ ) to have maximal or, at least, very large mixing? The conventional wisdom tells us that mixing angles are determined by lepton mass ratios. In the quark sector, the Cabibbo angle is well reproduced by the formula $\tan ^{2} \theta_{C}=$ $m_{d} / m_{s}$. In the same spirit, the neutrino mixing angle, in the case of two generations, is expected to be determined by the ratios $m_{e} / m_{\mu}$ and $m\left(\nu_{1}\right) / m\left(\nu_{2}\right)$. If $m\left(\nu_{1}\right) \ll m\left(\nu_{2}\right)$, one expects the neutrino mixing angle to be very small.

However, this conclusion is not valid when one invokes the see-saw mechanism. The possibility that the see-saw mechanism may enhance lepton mixing up to maximal was discussed in Refs. [29,30]. When the see-saw mechanism is invoked with more than one generation of neutrinos, the mixing angles for the resultant light Majorana neutrinos become dependent upon the masses of heavy right-handed Majorana neutrinos which are usually provided by GUTS models. In particular, when certain relationships among the mass parameters in the original Dirac and heavy right-handed Majorana mass matrices are satisfied, the mixing angles for the light Majorana neutrinos can substantially be enhanced, even in the case of the usual hierarchy $m\left(\nu_{1}\right) \ll m\left(\nu_{2}\right) \ll m\left(\nu_{3}\right)$.

A full analysis of the atmospheric neutrino problem with arbitrary 3 mixing angles will be given elsewhere.

\section{ACKNOWLEDGMENTS}

The authors would like to thank Jaeyoung Lee for his contributions in the early stage of this work. They would also like to express their gratitude to S. Bilenky, A. Bottino and Jewan Kim for many valuable discussions. This work was supported in part by the Research Funds of the Ministero dell'Università e della Ricerca Scientifica e Tecnologica and by the National Science Foundation, USA. 


\section{REFERENCES}

[1] S.M. Bilenky and B. Pontecorvo, Phys. Rep. 41, 225 (1978); S.M. Bilenky and S.T. Petcov, Rev. Mod. Phys. 59, 671 (1987).

[2] C.W. Kim and A. Pevsner, Neutrinos in Physics and Astrophysics, Contemporary Concepts in Physics, Vol. 8, (Harwood Academic Press, Chur, Switzerland, 1993).

[3] R. Davis Jr., Talk presented at the $6^{\text {th }}$ International Workshop on Neutrino Telescopes, Venezia, March 1994.

[4] K. S. Hirata et al., Phys. Rev. Lett. 65, 1297 (1990); Phys. Rev. D 44, 2241 (1991); Y. Suzuki, Talk presented at the $6^{\text {th }}$ International Workshop on Neutrino Telescopes, Venezia, March 1994.

[5] GALLEX Collaboration, Phys. Lett. B 285, 376 (1992); Phys. Lett. B 314, 445 (1993); Phys. Lett. B 327, 377 (1994).

[6] SAGE Collaboration, in Proc. of the $27^{\text {th }}$ Int. Conf. on High Energy Physics, Glasgow, July 1994.

[7] J.N. Bahcall and R. Ulrich, Rev. Mod. Phys. 60, 297 (1988); J.N. Bahcall, Neutrino Physics and Astrophysics, Cambridge University Press, 1989; J.N. Bahcall and M.H. Pinsonneault, Rev. Mod. Phys. 64, 885 (1992).

[8] H.H. Chen, Phys. Rev. Lett. 55 (1985) 1534; R.S. Raghavan, S. Pakvasa and B.A. Brown, Phys. Rev. Lett. 57 (1986) 1801; J.N. Bahcall, M. Baldo-Ceolin, D.B. Cline and C. Rubbia, Phys. Lett. B 178 (1986) 324; S. Weinberg, Int. J. Mod. Phys. A 2 (1987) 301; C. Rubbia, CERN-PPE/93-08; G. Fiorentini et al., Phys. Rev. D 49, 6298 (1994).

[9] S.M. Bilenky and C. Giunti, Phys. Lett. B 311 (1993) 179; Phys. Lett. B 320 (1994) 323; DFTT 12/94 (hep-ph@xxx.lanl.gov/9403345).

[10] K.S. Hirata, Phys. Lett. B 205, 416 (1988).

[11] K.S. Hirata et al., Phys. Lett. B 280, 146 (1992).

[12] Y. Fukuda et al., Phys. Lett. B 335, 237 (1994).

[13] R. Becker-Szendy et al., Phys. Rev. D 46, 3720 (1992); D. Casper et al., Phys. Rev. Lett. 66, 2561 (1992).

[14] C. Berger et al., Phys. Lett. B 227, 489 (1989); Phys. Lett. B 245, 305 (1990).

[15] M. Aglietta et al., Europhys. Lett. 8, 611 (1989); Europhys. Lett. 15, 559 (1991).

[16] T. Kafka, Nucl. Phys. B (Proc. Suppl.) 35, 427 (1994).

[17] G. Barr, T.K. Gaisser and T. Stanev, Phys. Rev. D 39, 3532 (1989); G. Barr, T.K. Gaisser, S. Tilav and D. Lipari, Phys. Lett. B 214, 147 (1988); W. Frati, T.K. Gaisser, A.K. Mann and T. Stanev, Phys. Rev. D 48, 1140 (1993); T.K. Gaisser, Nucl. Phys. B (Proc. Suppl.) 35, 209 (1994).

[18] L. Wolfenstein, Phys. Rev. D 17, 2369 (1978); Phys. Rev. D 20, 2634 (1979); S.P. Mikheyev and A.Yu. Smirnov, Yad. Fiz. 42, 1441 (1985) [Sov. J. Nucl. Phys. 42, 913 (1985)]; Il Nuovo Cimento C 9, 17 (1986).

[19] V. Barger, R.J.N. Phillips, and K. Whisnant, Phys. Rev. Lett. 69, 3135 (1992); P.I. Krastev and S.T. Petcov, Phys. Lett. B 299, 99 (1993); Phys. Rev. Lett. 72, 1960 (1994).

[20] GALLEX Collaboration, Phys. Lett. B 285, 390 (1992); X. Shi, D.N. Schramm and J.N. Bahcall, Phys. Rev. Lett. 69, 717 (1992); Phys. Rev. D 47, 2220 (1993); N. Hata and P.G. Langacker, Phys. Rev. D 50, 632 (1994); L.M. Krauss, E. Gates and M. White, 
Phys. Lett. B 299, 94 (1993); Fermilab-Pub-94/176-A (hep-ph@xxx.lanl.gov/9406396); G.L. Fogli and E. Lisi, Astropart. Phys. 2, 91 (1994); G. Fiorentini et al., Phys. Rev. D 49, 6298 (1994).

[21] T.K. Kuo and J. Pantaleone, Phys. Rev. Lett. 57, 1805 (1986); Phys. Rev. D 35, 3432 (1987); C.W. Kim and W.K. Sze, Phys. Rev. D 35,1404 (1987); A. Bottino, J. Ingham and C.W. Kim, Phys. Rev. D 39, 909 (1989); G.L. Fogli, E. Lisi and D. Montanino, Phys. Rev. D 49, 3626 (1994); Talk presented at the $6^{\text {th }}$ International Workshop on Neutrino Telescopes, Venezia, March 1994.

[22] A. Acker, J.G. Learned, S. Pakvasa and T.J. Weiler, Phys. Lett. 298B, 149 (1993).

[23] S. Nussinov, Phys. Lett. 63B, 201 (1976).

[24] L. Wolfenstein, Phys. Rev. D 18, 958 (1978).

[25] G.S. Vidyakin et al., Zh. Eksp. Teor. Fiz. 98, 764 (1990) [Sov. Phys. JETP 71, 424 (1990)].

[26] G. Zacek et al., Phys. Rev. D 34, 2621 (1986).

[27] J.N. Bahcall and M.H. Pinsonneault, Rev. Mod. Phys. 64, 885 (1992).

[28] M.B. Aufderheide et al., Phys. Rev. C 49, 678 (1994).

[29] A.Yu. Smirnov, Phys. Rev. D 48, 3264 (1993).

[30] C.W. Kim, in Proceedings of the $3^{\text {rd }}$ Nestor International Workshop, October 1993, Pylos, Greece, edited by L.K. Resvanis. 


\section{FIGURES}

FIG. 1. Separate fits of the sub-GeV (a) and multi-GeV (b) data for several different values of $\Delta m_{32}^{2}$

FIG. 2. Values of $\chi^{2}$ for the separate fits of the sub-GeV (a) and multi-GeV (b) data and the combined fit (c).

FIG. 3. Solar neutrino event rates as functions of $\Delta m_{21}^{2}$. The horizontal solid lines are the central values of the experiments with the dashed lines representing one standard deviation. The oscillating solid lines represent the theoretical values, with the dotted lines indicating one standard deviation resulting from the errors in the SSM. 
This figure "fig1-1.png" is available in "png" format from: http://arxiv.org/ps/hep-ph/9411219v4 
This figure "fig1-2.png" is available in "png" format from: http://arxiv.org/ps/hep-ph/9411219v4 
This figure "fig1-3.png" is available in "png" format from: http://arxiv.org/ps/hep-ph/9411219v4 


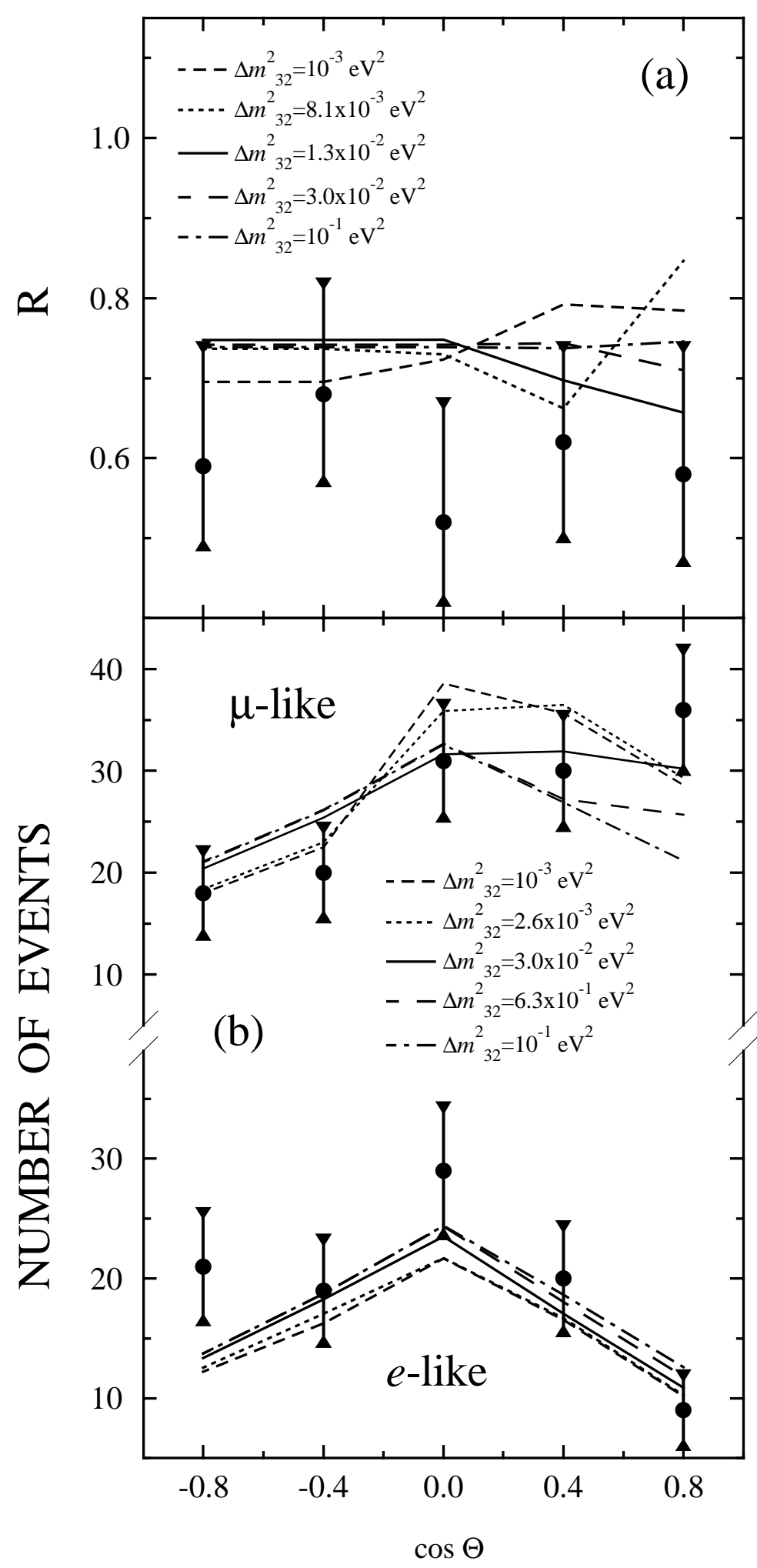

FIG. 1. Separate fits of the sub-GeV (a) and multi-GeV (b) data for several different values of $\Delta m_{32}^{2}$. 


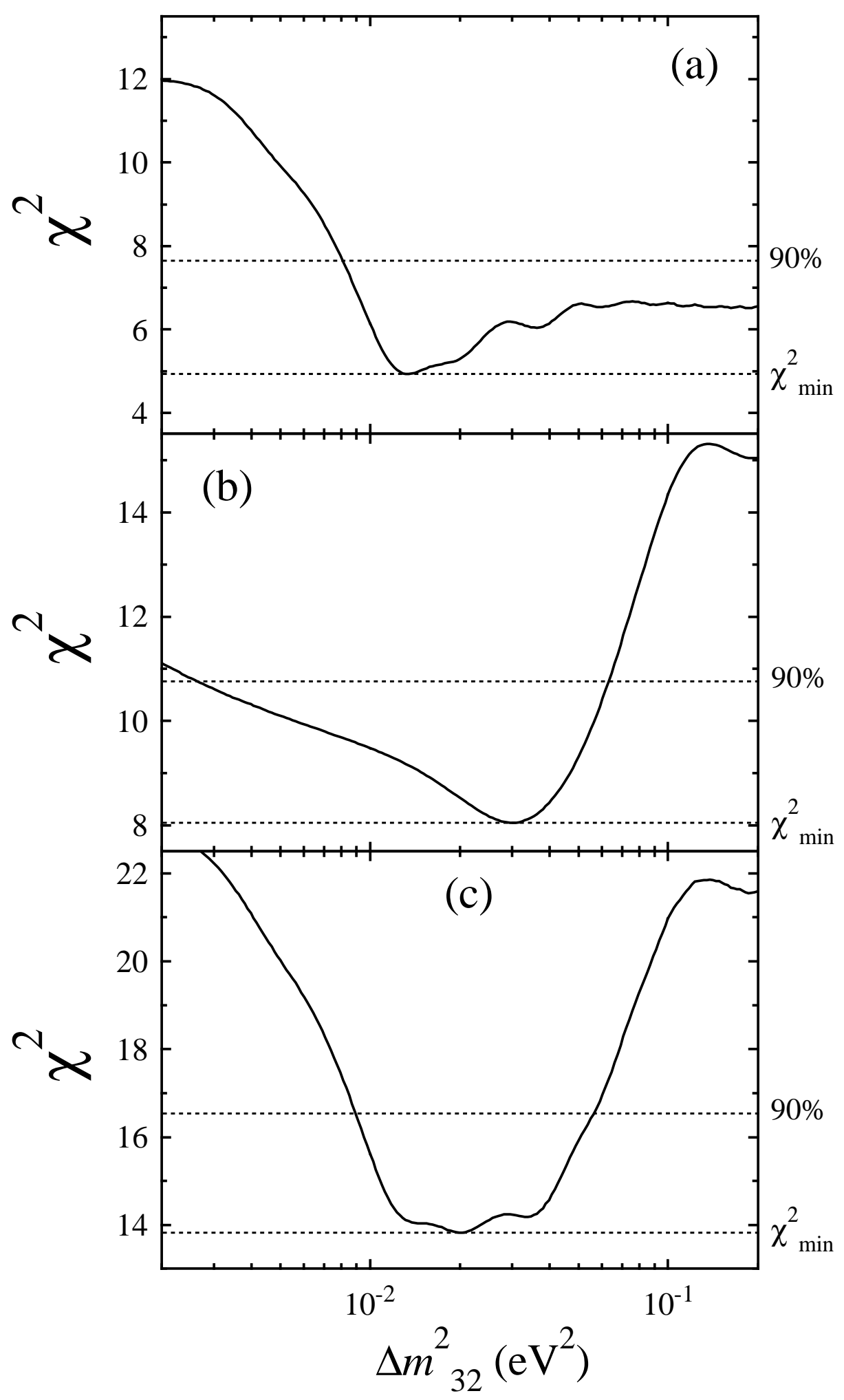

FIG. 2. Values of $\chi^{2}$ for the separate fits of the sub-GeV (a) and multi-GeV (b) data and the combined fit (c). 


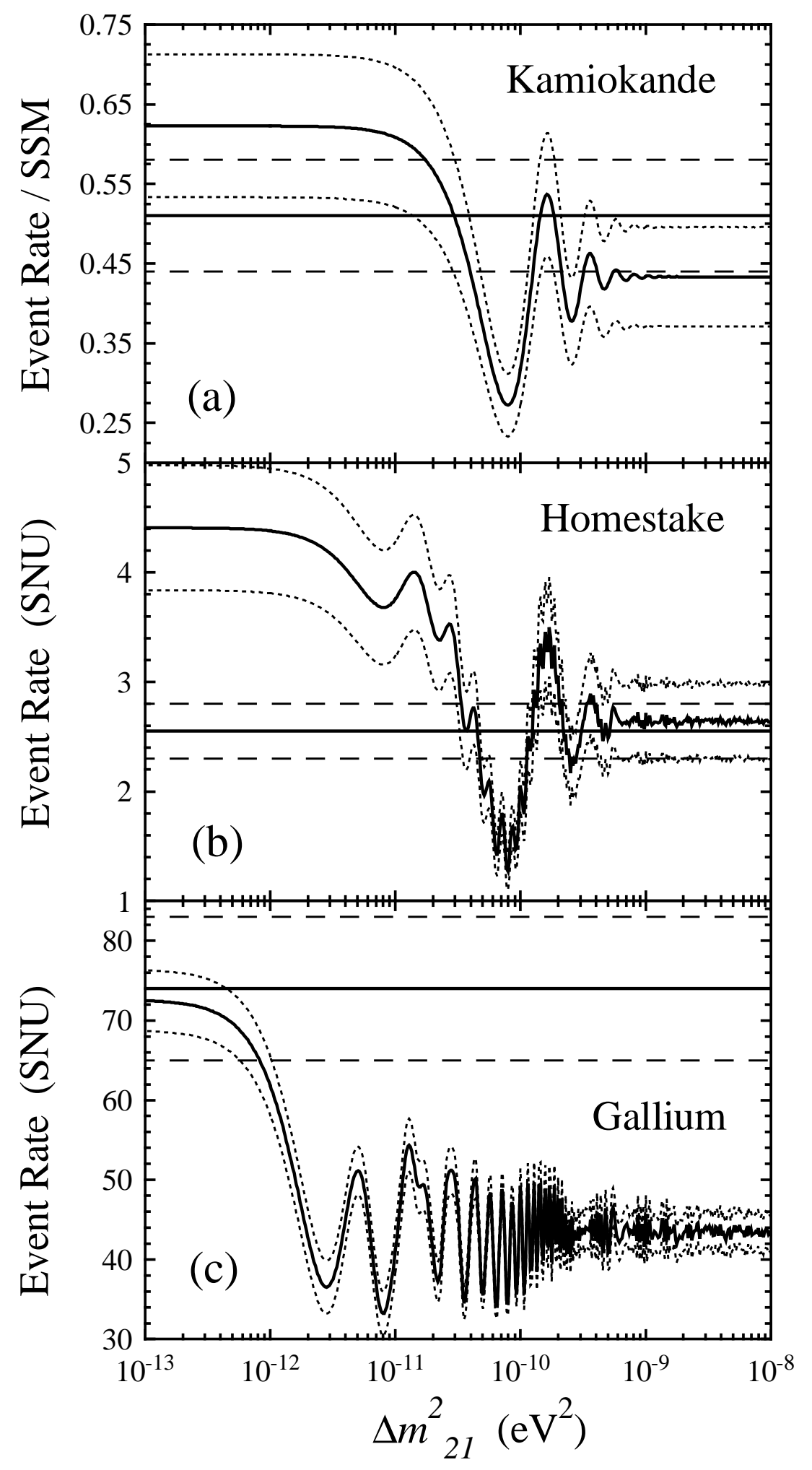

FIG. 3. Solar neutrino event rates as functions of $\Delta m_{21}^{2}$. The horizontal solid lines are the central values of the experiments with the dashed lines representing one standard deviation. The oscillating solid lines represent the theoretical values, with the dotted lines indicating one standard deviation resulting from the errors in the SSM. 